\title{
Development of web-based surveillance system for Internet of Things (IoT) application
}

\author{
Siti Noorjannah Ibrahim, A. H. Hasan Basri, Ani Liza Asnawi \\ Department of Electrical and Computer Engineering, Kulliyyah of Engineering, \\ International Islamic University Malaysia, Malaysia
}

\begin{tabular}{l} 
Article Info \\
Article history: \\
Received Feb 10, 2019 \\
Revised May 12, 2019 \\
Accepted May 27, 2019 \\
\hline Keywords: \\
Internet of Things (IoT) \\
Live video streaming \\
Node-red tool \\
Raspberry Pi \\
Web-based surveillance system
\end{tabular}

\section{Article Info}

Article history:

Received Feb 10, 2019

Revised May 12, 2019

Accepted May 27, 2019

Web-based surveillance system

\begin{abstract}
With the advancement of wireless technology, our dependency on smart system has increases to a higher level than before. Without doubt, integration between different technologies becomes inevitable in order create affordable surveillance system. This paper presents the development of with web-based surveillance system with a dedicated Android-based mobile application using a Raspberry Pi and its supporting components i.e., Pi-Camera, PIR motion sensor, Ultrasonic sensor, web-based \& mobile application. The designed system also utilizes Node-Red development tools as the platform to integrate all components of the system, MQTT as the communication protocol for data acquisition and ThingSpeak as the middleware. The proposed system can be implemented over the internet using any computer and mobile devices, at anywhere and anytime. The system can automatically stream live video viewed from the Android mobile application and the Raspberry Pi device can send an alert notification to users via email and SMS. The system can be one possible features in smart home system and is considered as an affordable solution, customizable and easy to implement in comparison with other commercial surveillance system products such as CCTV or IP Camera.
\end{abstract}

Copyright (C) 2019 Institute of Advanced Engineering and Science. All rights reserved.

Corresponding Author:

Siti Noorjannah Ibrahim,

Department of Electrical and Computer Engineering,

Kulliyyah of Engineering,

International Islamic University Malaysia, Malaysia.

Email: noorjannah@iium.edu.my

\section{INTRODUCTION}

The risen of crime cases in Malaysia particularly in big cities like Kuala Lumpur, has heighten the necessity in developing a sophisticated surveillance system by integrating the current surveillance system with the internet for continuous monitoring of activities; at anywhere, at any time. The growth of technologies, especially in the field of Internet of Things (IoT) can enhance basic functionalities of a surveillance system. Surveillance comes from a French word meaning "watching over" [1], and with the advancement of the hardware and software in recent years, surveillance system has rapidly improved from a "Dumb camera", CCTV and integrated with other sensors for a multifunctional system which can be made available at a low cost, depending on the system components and features.

In retrospect, the first-generation surveillance systems (1960s-1980s) are mainly human-operated system where the operators examine, classify and view the issue of video stream captured as analog signals that are transmitted to a remote-control room and displayed on large screens. The second-generation (2G) surveillance systems in 1980s-2000s, started using digital video communications and utilized automatic video processing method to assist operator in detecting some simple suspicious events. IP cameras also known as the network cameras were introduced in the $2 \mathrm{G}$ system. From the year 2000 onwards, many surveillance systems can be integrated with multiple sensors such as infrared, audio, thermal etc. using a processing unit of controllers that can perform image processing and relay multiple inputs simultaneously. 
Thus, a human operator can detect and describe strange situations from the amount of information displayed [2].

Current surveillance systems can utilize multiple sensors integration with sensors together with one or more imaging techniques i.e., infrared, visible, or thermal, and could also include a reconfigurable processing component (FPGA, DSP), a power element (Solar, Battery), communication unit (Wi-Fi, 4G,3G) and storage module. Nevertheless, a basic surveillance system consists of three essential items: the front-end video capturing unit, the central control system unit and the end-user interface unit. The video capturing unit acts as the "eye" of the system, utilizes camera and/or digital video recorder (DVR) to capture videos or images. The central control system relays captured videos from the camera to a storage system (to local or cloud storage) by applying certain image processing technique for video compression and delivers them to the end-user interface unit.

A surveillance system can be categorised into centralized or distributed system. In the former system, captured videos is processed at the central server unit while the latter, videos are processed at the embedded controller units and distributed through camera channels before saved in the server. There are several types of the surveillance system available in the market: Closed Circuit Television (CCTV) camera and Internet Protocol (IP) camera. The CCTV system requires continual monitoring of every action which is not at ease, makes utilization of CCTV camera are expensive. Meanwhile, the operation of IP camera is also quite costly and could cause a huge problem when it becomes open to hackers via internet while watching for the cameras [3]. A reliable storage for saving captured videos is crucial to ensure the safe keeping of data of the surveillance system. Furthermore, tracing back past events through captured videos is one of the vital functions for the system because surveillance videos could be the main evidence for any crime scene.

\subsection{IoT and web-based surveillance system}

The "Internet of Things" phrase comes from two words: the "Internet" and "Things" that defines the interactions or communications between "Things" and "Human" or among "Things" through the availability of a structured computing system connected worldwide through networks using Transmission Control Protocol/ Internet Protocol (TCP/IP) as the common reference interface. It gives us the meaning that both non-living things such as industrial machinery or home appliances and living things such as human can interacts through a common protocol via internet. Web-based concept or known as cloud computing and Internet of Things (IoT) became part of our lives and the concept is expected to grow further known as the Cloud IoT [4].

Therefore, a real-time IoT application must implement the Application Programming Interfaces (API) or Service Oriented Architecture (SOA) components in its development to allow web-services and other devices to interact with each other [5]. When expanding the idea of "Web-Based application" or a software as the embedded domain, the communication with "Things" such as reading a sensor or writing data must be through the "actors". "Actors" can be characterized as sensors, actuators and control system. The communication between actors is executed using ports. The perception of web-based SOA is often referred to as ad-hoc request-response message design and control which uses natural event/data-driven. The information is collected by sensors and distributed to the connected services. These connected services can be actuator facilities generating a hardware operation or control services which can produce output or information based on their inputs.

Node-Red Integrated Development Environment (IDE) is an open source programming tool and has becomes one of the common operative tool in developing IoT applications. The IDE allows wiring of hardware devices, APIs and online services on the same platform. It offers process-flow-based development tools which makes the process of developed IoT applications easy with minimal programming codes. It uses the concept of node-to-node to create network by wiring graphical blocks and functions. Node-Red supports microcontroller such as Raspberry Pi, Arduino as well as Windows [6-7]. In other words, a developer can structure the flow of data acquisition, monitoring, transportation, and analytics of any IoT applications using a single platform. The platform can also link developers with social network platforms such as Twitter, Telegram, Facebook, thus the deployment of IoT applications become easier with various functions and crosslinks between different platforms.

MQTT or the Message Queuing Telemetry Transport is an IoT communication protocol used for data acquisition. It uses a low power supply that consists of minor data packet size which runs over TCP/IP [8]. There are two core software mechanisms in MQTT protocol: 1) MQTT client and 2) MQTT broker. MQTT client is a device i.e., Windows, Raspberry Pi, Arduino that uses JavaScript code. Meanwhile, MQTT broker works as the publisher or subscriber of data. The web platform that supports MQTT broker are HiveMQ and mosquitto [8]. MQTT features a topic (UTF-8 string) and Quality of service (QoS). A topic functions to filter messages from any connected clients for example from the subscriber to the publisher. The topic is controlled by MQTT broker. As for QoS, it functions to the assurance of message delivery. 
It has three level of QoS which are QoS 0, QoS 1, and QoS 2. QoS 0 function to transmit the message only once. QoS 1 function to issue a message at least once and finally QoS 2 function to validate sending a message is received by the publisher [9]. So, by applying the MQTT in an IoT applications, the communications between sensor, sensor nodes, microcontrollers and the internet can be more reliable, more efficient (in distributing information) with low power consumption.

ThingSpeak is an Internet of Things middleware and one of the common Application Programming Interfaces or APIs web platform used for building IoT applications. ThingSpeak is compatible with microcontrollers such as Raspberry $\mathrm{Pi}$ and Arduino. It offers many features such as storing/receiving data and analyzing data on visual graphs for IoT applications [10]. The data collected from microcontroller can be visualised in ThingSpeak channel. It also can link with MQTT as the broker. Thus, in Thing Speak, developers can monitor data logging from sensors.

An Arduino based (Atmega8) security system was designed in [6]. It uses an open source electronic platform to program the system. The Atmega8 board relays data from the ultrasonic sensor to a serial monitor when any movements are detected. Then, the system alerts users through SMS sent via GSM module. In [11], a smart surveillance system using PIR sensor and GSM network was developed. The system comprises of PIR sensor and video camera integrated using a peripheral interface controller (PIC 16F877A). The PIC controls all components that connected to it. Some benefits of using PIC in a surveillance system include easy to program, low in cost, and compatible with other microcontrollers.

A smart security system and monitoring system using a Raspberry Pi and PIR sensor has been demonstrated in [12]. Raspberry $\mathrm{Pi}$ is equipped with GPIO pins that allows interface with various sensors [13]. In this system, the Open Source Computer Vision (OpenCV) software which runs on the Raspbery Pi board, performs an image processing of videos captured from the camera, control the alert algorithm and send a message to the user. The system also able to control other function such as motion detectors on the same platform.

This paper presents the development of a surveillance system that allows people to monitor their homes, at anytime and anywhere. The aim of this work is to design and develop an affordable CCTV-like surveillance system accessible via the internet using a dedicated website and mobile application (mobile apps) using a Raspberry Pi microprocessor. The system allows easy implementation at home as users can get connected to the surveillance system from any computers or mobile devices such as laptops, tablets or mobile phones through the website and application. Hence, the system offers an alternative for storing captured videos from the surveillance system. It discovers a new technique for relaying captured videos through the internet via MQTT broker to communicate to the middleware. Here, we use two middleware solutions: Noderack and ThingSpeak, just for comparison. With this structure, we can counter the problem of surveillance system without saving the recorded videos in a storage but still capable to analyse live incident from the videos [3].

Technically, this surveillance system comprises Raspberry Pi microprocessor, Pi-Camera with mounted servo-motor, PIR motion sensor, Ultrasonic sensor, buzzer and LED. The Passive Infrared sensor (PIR) is useful in a low-cost surveillance system through which it provides infrared radiation in detecting human presence from human body temperature. The system is programmed with a web-based and mobile app for its management and configured for internet connection which enables remote monitoring by controlling the movement of the camera, and then simultaneously record the image of the home intruder into the system storage and send an alert message via email and/or SMS to users. Unlike commercial CCTV system, the system allows user to view the live streaming video through an Android-based mobile application in the which can reduce the cost of the system.

\section{RESEARCH METHOD}

The designed surveillance system features an alarm unit, alert notification to users unit, camera movement control unit and data acquisition unit. It comprises of Raspberry Pi 3 Model B, Pi-Camera module 5MP, PIR sensor, Ultrasonic sensor, Piezo buzzer and Servo motor. The components were programmed using OpenCV, connected Node-Red and integrated with internet via a mobile application through a dedicated website to create a workable surveillance system as shown in Figure 1. The Raspberry Pi controls all the operation of sensors, Pi-Camera and data acquisition for the whole system.

The first operation is to detect intruder and trigger the alarm unit. Here, the PIR sensor detects any movements within the vicinity of two centimeters to six meters. The second stage is the notification operation. When PIR sensor is in the state of active or HIGH, the system will automatically trigger the camera to function and capture the image of intruder. The captured image is then stored in SD card of the Raspberry Pi. Simultaneously, two alert notifications will be sent to user via SMS and email with the captured image as an attachment. 


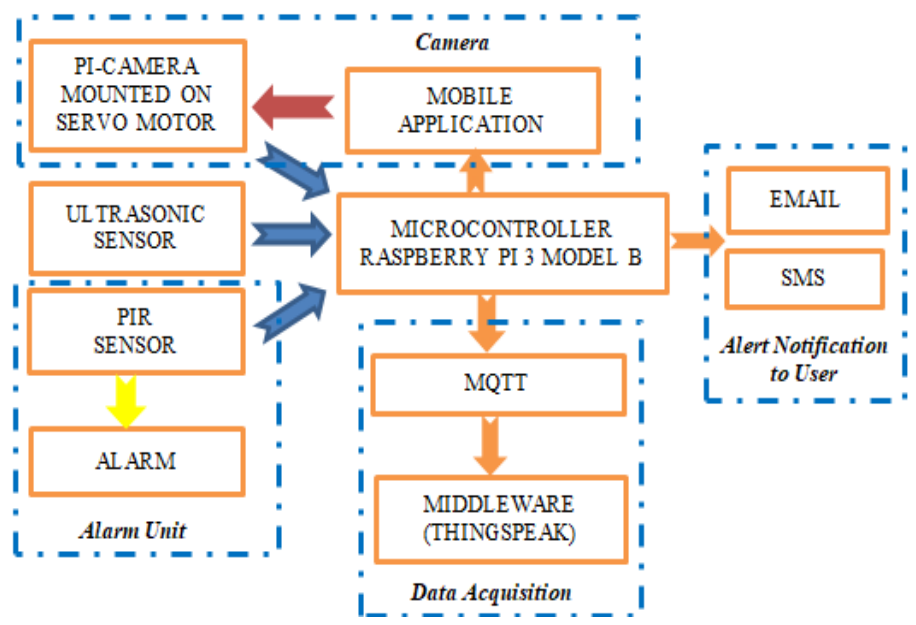

Figure 1. The designed surveillance system equipped with an alarm unit, data acquisition, camera movement and alert notification functions

This surveillance system also allows users to view the live streaming video through a dedicated Android mobile application after getting the notification alert. When the detection of intruder happened, the system will remain active for 10 minutes. If the motion of intruder is detected, the camera will automatically $\mathrm{ON}$ and record the live streaming video. During that period of time, users can remotely monitor live incident through an android mobile application that has camera movement feature i.e., to rotate left and/or right. The program then will remain in video mode until the sensor goes to idle mode (0) which is after 10 minutes. When the sensor in idle mode (0), it will start to sense movement again through PIR sensor. With this alternating mode, only live streaming in 10 minutes duration will be recorded to safe the storage space and bandwidth.

Subsequently, Raspberry Pi acquires data form the ultrasonic sensor that consist of distance between the intruder and the surveillance box and relays the data to the MQTT broker. The data is subscribed by MQTT broker and then publish to the MQTT clients i.e., Node-Red and Thing Speak. In the node-red dashboard, the ultrasonic sensor distance graph will be displayed. Distance graph also can be viewed by users through Thing Speak channel. The flow chart showing the overall operations of the designed surveillance system is depicted in Figure 2.

\subsection{Node red flow and MQTT broker}

Node-Red is a programming tool used to implement of the Internet of Things (IoT) [14]. It is a websitebased platform that makes it easy for wiring all hardware components and have a wide range of palette node. With Node-red, a programmer could do data acquisition, monitoring, transportation, and analytics. It also has an app feature to observe home that can be developed by the developer. It also has an advantage in term of using small amount programming code and it is compatible with a variety of other platforms such as Thing Speak, MQTT, and IBM Watson.

The PIR sensor is connected to output buzzer and LED in Node-Red as shown in Figure 3. The connection is set up using a Raspberry-Pi GPIO pin. It is then linked to a function of the PIR sensor to check whether there is a movement of an intruder, PIR goes high (1), thus buzzer and LED also goes high (1). Next, function node is joined to produce an output of text which displays "intruder detected!" and output audio that present voice if motion detected. Function node also connected to the camera so that when PIR goes high (1), it automatically captures an image of the intruder and send it to email and SMS notification.

In this project, we use Twilio platform to send SMS alert. But, Twilio node need to be connected to the function of activity. Then, the image of intruder also will be saved to file in the raspberry pi SD card. As for MQTT part, the ultrasonic sensor is connected to the output in debug node, to the chart which displays in the node-red dashboard and to subscribe to input MQTT broker [9]. MQTT broker is connected to the same Wi-Fi connection as surveillance box. The MQTT broker node also needs to be inserted topic and quality of service (QoS). For this project, the topic for MQTT broker is distance. Then the QoS was set to level 2 to make sure the message is received by the client. Next, MQTT publish node is connected to the ThingSpeak channel where the channel received distance data every time there is human detection. At the same time, button node is used to trigger the rotation of servo motor. The button is to make users easily to press it when viewing live stream video. Lastly, template node is used to write functional code for live stream video. 


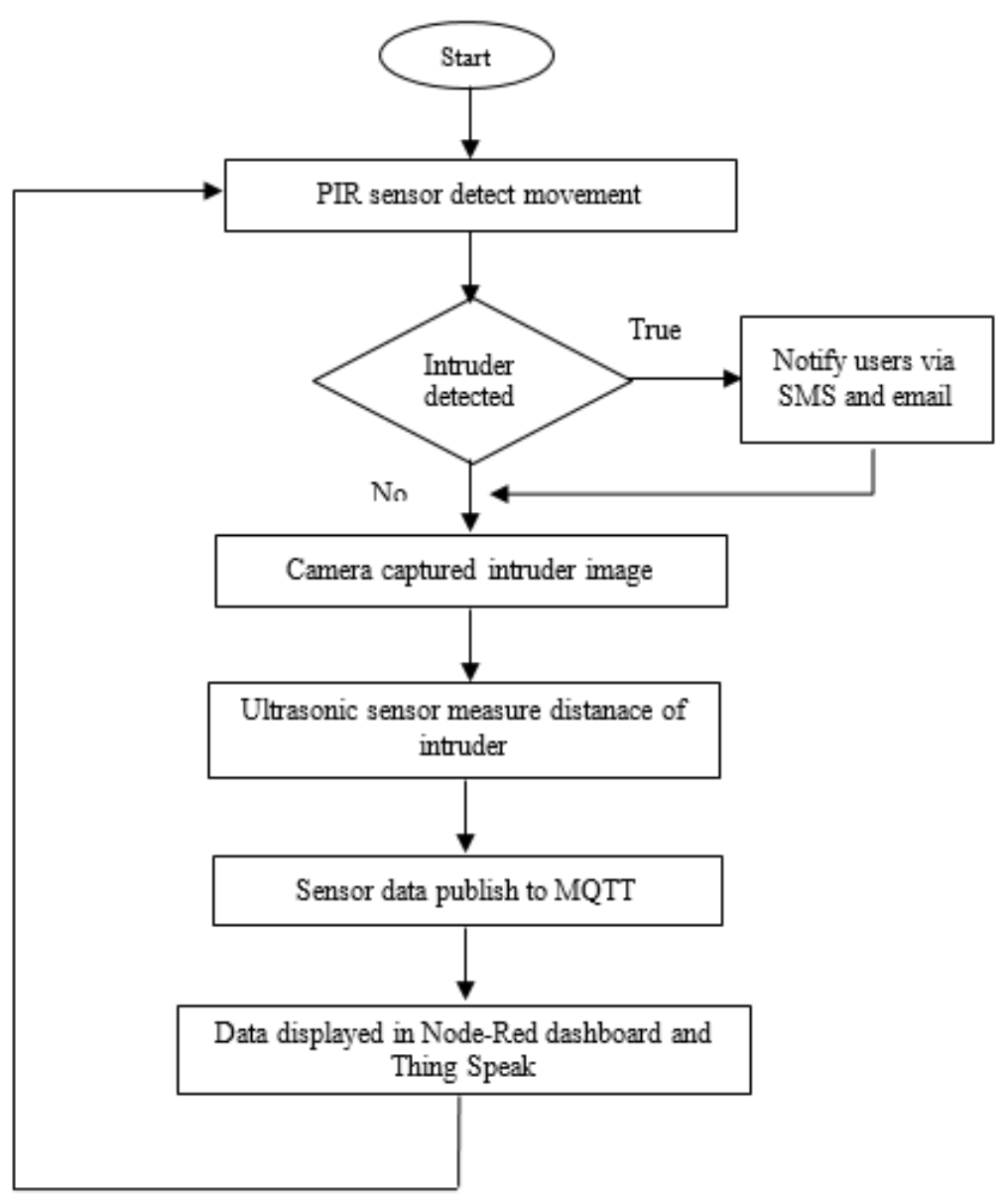

Figure 2. Flowchart of the surveillance system with data acquisition

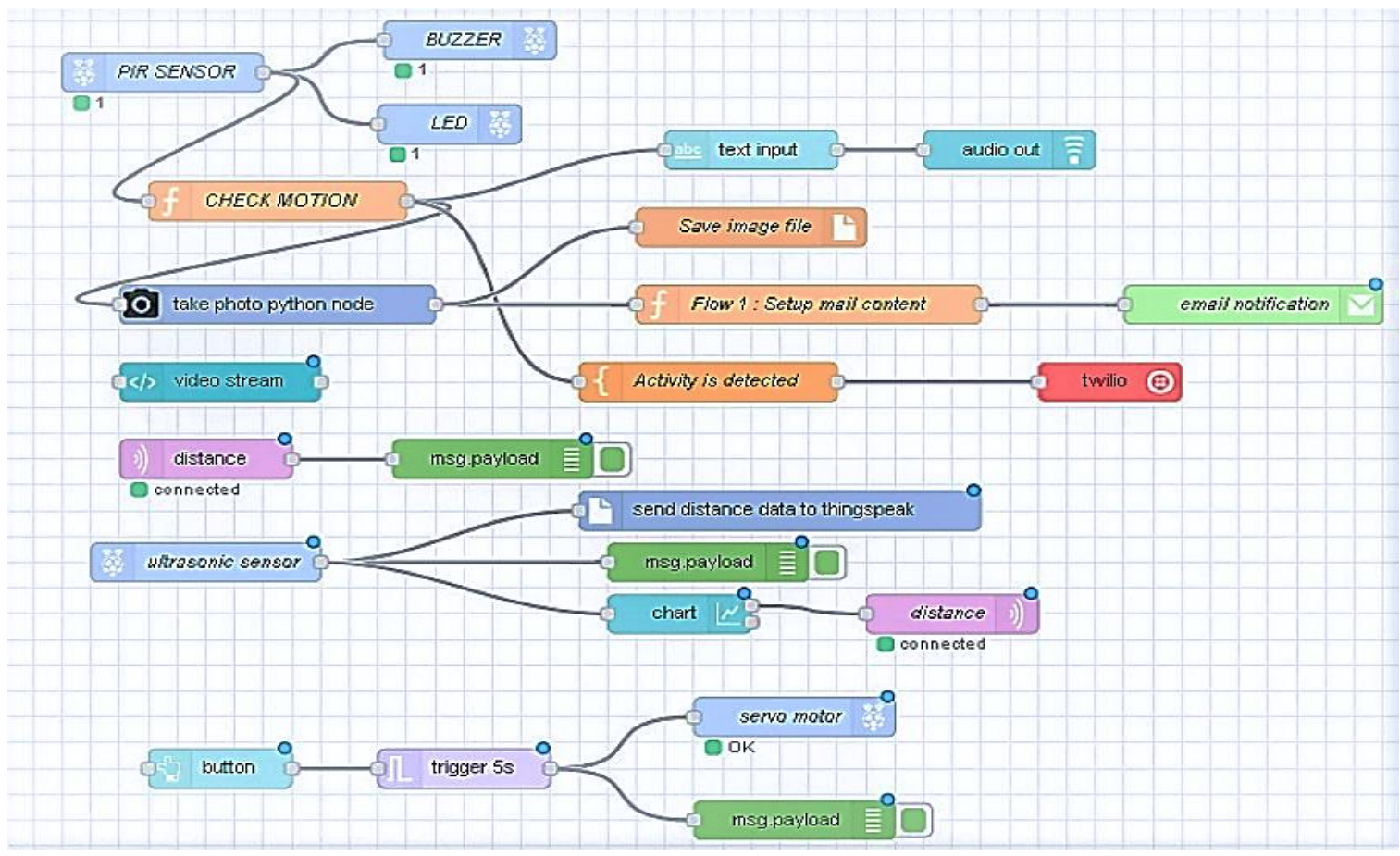

Figure 3. Process flow and connections between sensors of the designed system in node-red 


\subsection{Android application using APK builder}

Mobile Application is created using the Website 2 APK builder Software as shown in Figure 4. The software is a Java based application designed to convert online HTML5 website to the mobile application. It has the advantage of Google Play Ready Apps and Java Runtime Environment [15]. In this surveillance, the system linked Node-Red dashboard with the software by inserting the Node-Red dashboard URL address.

The complete setup of the surveillance system comprises of a camera was placed on top of the box mounted on a servo motor as shown in Figure 5. Meanwhile, the PIR and ultrasonic sensors are placed next to each other as the main part of the system to detect motion and to calculate distance between the surveillance system and the intruder. At the bottom of the box, buzzer (alarm) and LED are being presented to notify output from the sensors.

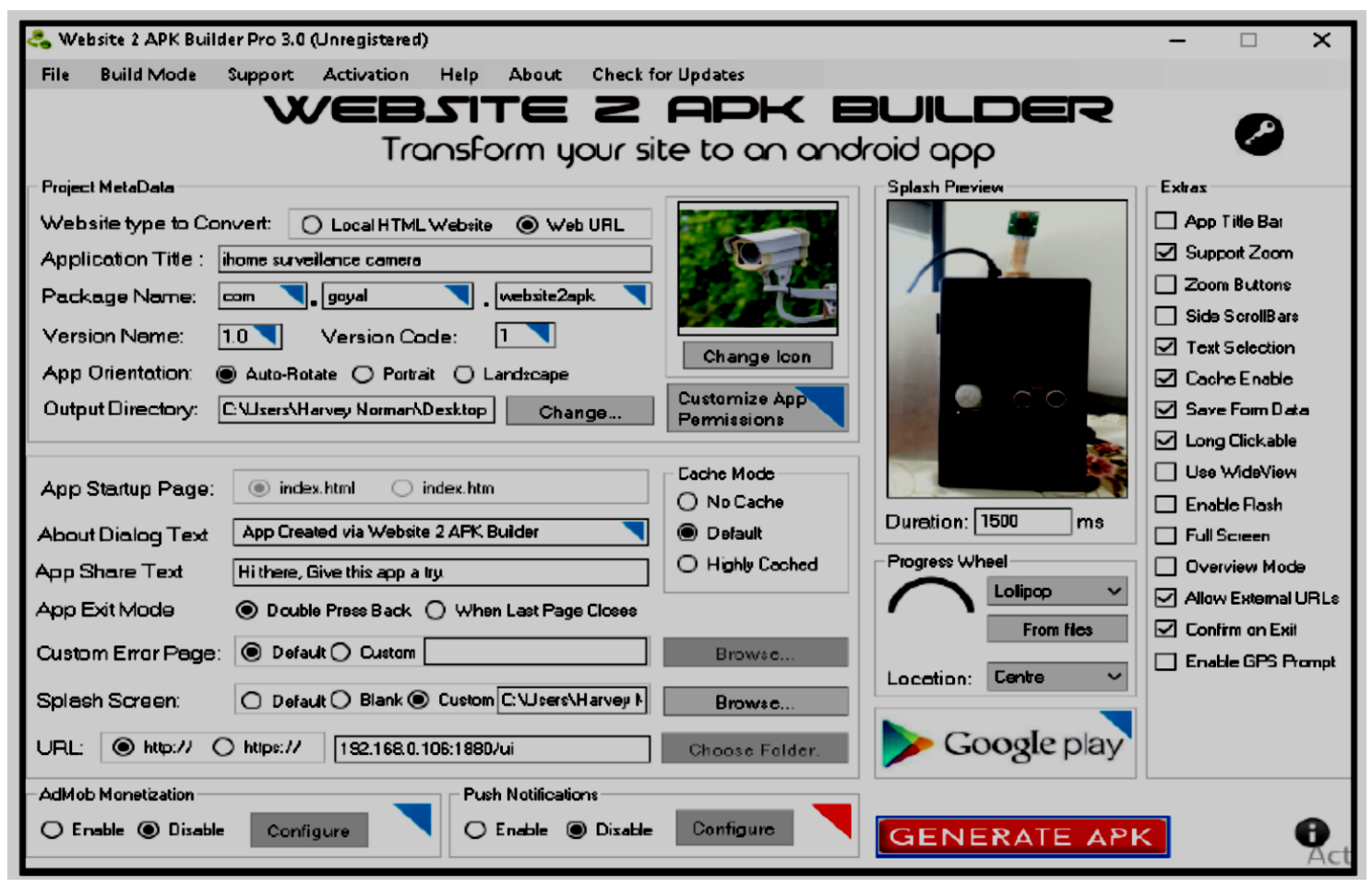

Figure 4. The website to APK builder menu

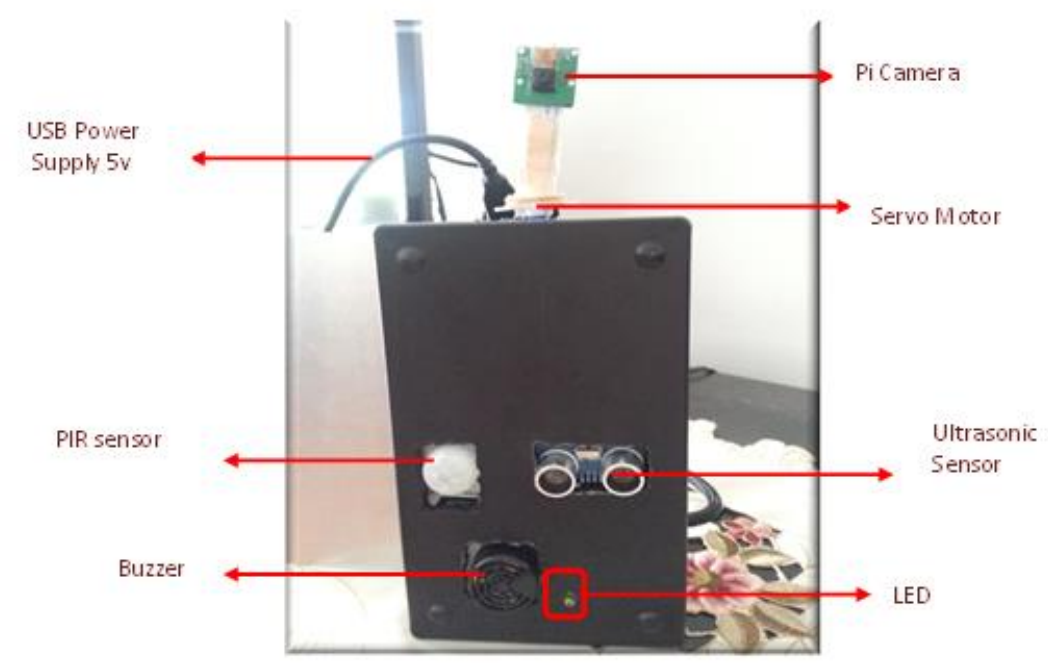

Figure 5. The complete setup of the surveillance system 


\section{RESULTS AND ANALYSIS}

\subsection{Sensors and system testing}

At first, were tested to determine the accuracy of ultrasonic sensor. Table below shows accuracy and error distance by ultrasonic sensor. It can be inferred that there is a difference between actual distance and distance measured by ultrasonic sensor and the differences are defined as distance error. The percentage of distance error is the percentage of difference between the actual distance and the ultrasonic distance i.e., actual distance - the ultrasonic distance $x 100 \%$. From observations, it can be incurred that the distance error are the effects of interference and noise from signals propagated and reflected by the environments or changes in room temperature [5]. This is one of the limitations when using low-cost ultrasonic sensor. Another apparent drawback is the distance error will increase as the distance of object increases particularly when involving moving objects as shown in Table 1.

Table 1. Distance measured using ultrasonic sensor and distance error

\begin{tabular}{ccc}
\hline Actual distance $(\mathrm{m})$ & Ultrasonic sensor distance $(\mathrm{m})$ & Distance error $(\%)$ \\
\hline 1.00 & 0.93 & 7.0 \\
2.00 & 2.09 & 4.5 \\
3.00 & 3.77 & 25.7 \\
4.00 & 4.97 & 24.3 \\
\hline
\end{tabular}

\subsection{Android application integration}

The surveillance system provides an Android mobile application for users to monitor their home anywhere they go. The newly design mobile application was developed using Website 2 APK builder software tool. The application features a live streaming video with a button on a rotation camera to left and right as well as the status of the intruder as shown in Figure 6. In this experiment the image was captured in dim light (low light exposure) to observe the Pi-Camera ability at night. Results obtained indicate that the Pi-Camera managed to capture image of the intruder but lacking in distinguishable features such face, skin colour, etc. because of the low exposure of light. Due to this limitation, it can be inferred that the system can be improved by replacing Pi-Camera with other high resolution camera that equipped with night vision feature.

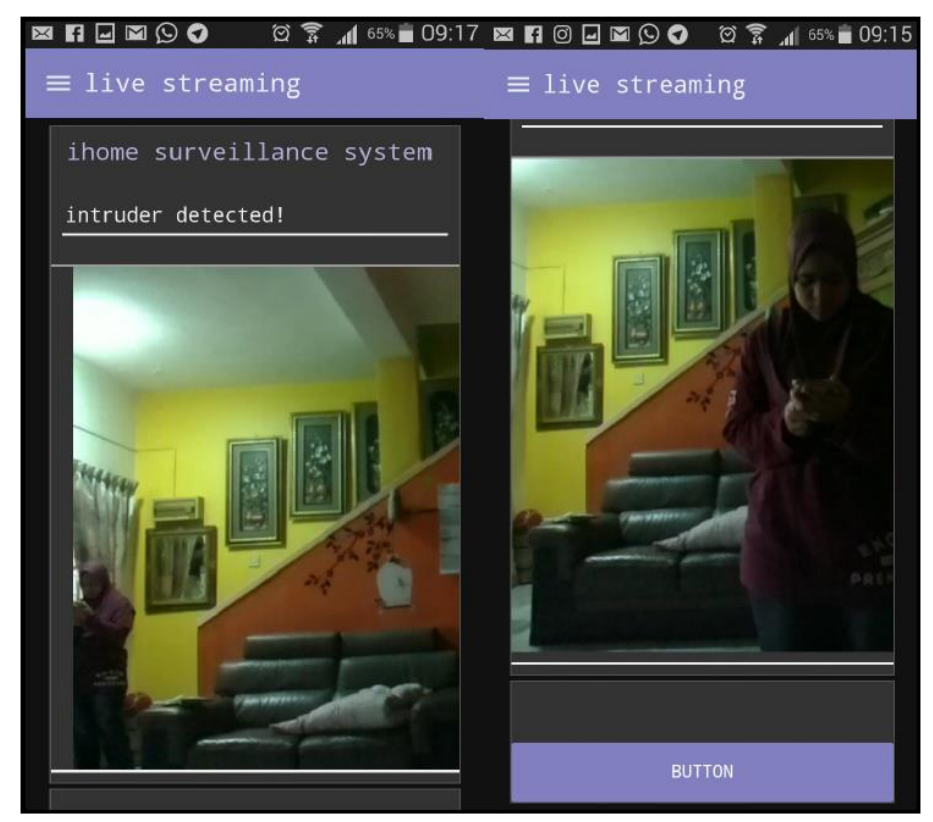

Figure 6. Live streaming video from the IP camera can be viewed from the newly developed Android application integrated with the surveillance system 


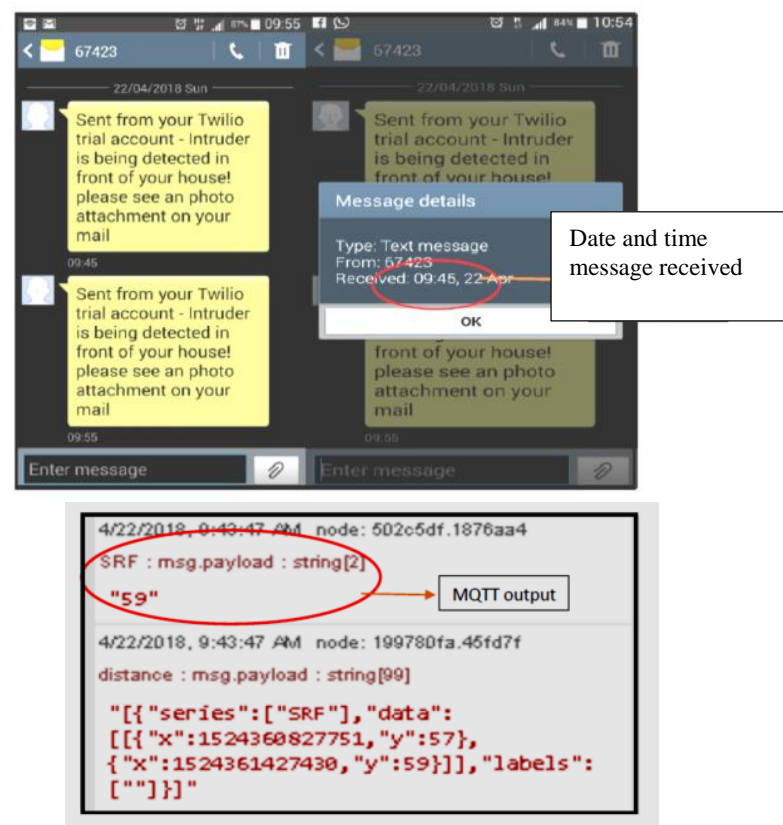

Figure 7. SMS notification to users and MQTT output

\subsection{SMS and email notifications}

Alert notifications to users via SMS and email will be transmitted from the surveillance system spontaneously when the PIR sensor detects any movement at the vicinity of the system. The SMS will alert users about the situation while the image of the intruder can be viewed through email. An example of the SMS notification is shown in Figure 7. Noticed that on the same day, SMS received by the user automatically notify after intruder detected at 9:45 am but it indicates the same time at 9:43 am which MQTT received distance data. This is because the user's mobile phone has set time 2 minutes earlier than the laptop. The results from Ultrasonic sensor were plotted in two platforms; Node-Red and ThingSpeak and have been discussed in [15] and the comparison between graph in the Node-red dashboard with the graph in ThingSpeak can be viewed in [15].

\section{CONCLUSION}

The Web-Based and mobile app surveillance system objectives have been achieved. The idea of creating an affordable prototype of IoT surveillance system has been successfully implemented using NodeRed and Thing Speak as the middleware. The system also has successfully sent alert notification via SMS and email to notify home users on any intrusion. However, the system can be improved by using high resolution camera with night vision feature to enhance its live streaming operation. Therefore, future improvements and enhancements are needed to be implemented in the system so that it can be a very valuable product for home security system.

\section{ACKNOWLEDGEMENTS}

The author would like to acknowledge the International Islamic University Malaysia for sponsoring a part of the project under Research Initiative Grant Scheme (RIGS 16-064-0228).

\section{REFERENCES}

[1] Narkhede YV, Khadke SG. Application of Raspberry Pi and PIR Sensor for Monitoring of Smart Surveillance System. International Journal of Electronics, Electrical and Computational System IJEECS. 2016 May;5(5):145-8.

[2] Deshmukh A, Wadaskar H, Zade L, Dhakate N, Karmore P. Webcam based intelligent surveillance system. Res Inventy: Int J Eng Sci. 2013;2(8):38-42.

[3] Ali AS, Al-Zawawi SA. The Effectiveness of Closed-Circuit Television (CCTV) In Malaysian Commercial Buildings. 
[4] Nguyen HQ, Loan TT, Mao BD, Huh EN. Low cost real-time system monitoring using Raspberry Pi. In2015 Seventh International Conference on Ubiquitous and Future Networks (ICUFN) 2015 Jul 7 (pp. 857-859).

[5] Botta A, De Donato W, Persico V, Pescapé A. On the integration of cloud computing and internet of things. InFuture internet of things and cloud (FiCloud), 2014 international conference on 2014 Aug 27 (pp. 23-30). IEEE.

[6] Yadav, G., Cse, B. T., Year, I. V, College, C. V. R., \& Devi, H. M. S. (2017). Arduino based Security System - An Application of IOT, (April), 209-212.

[7] Chaczko Z, Braun R. Learning data engineering: Creating IoT apps using the node-RED and the RPI technologies. InInformation Technology Based Higher Education and Training (ITHET), 2017 16th International Conference on 2017 Jul 10 (pp. 1-8). IEEE.

[8] Prabaharan J, Swamy A, Sharma A, Bharath KN, Mundra PR, Mohammed KJ. Wireless home automation and security system using MQTT protocol. InRecent Trends in Electronics, Information \& Communication Technology (RTEICT), 2017 2nd IEEE International Conference on 2017 May 19 (pp. 2043-2045). IEEE.

[9] Atmoko RA, Riantini R, Hasin MK. IoT real time data acquisition using MQTT protocol. InJournal of Physics: Conference Series 2017 May (Vol. 853, No. 1, p. 012003). IOP Publishing.

[10] Maureira MA, Oldenhof D, Teernstra L. ThingSpeak-an API and Web Service for the Internet of Things. Retrieved7/11/15World WideWeb, http://www. Mediate chnology. leiden. edu/images/uploads/docs/wt2014_thing speak. pdf. 2011.

[11] Sathishkumar M, Rajini S. Smart surveillance system using pir sensor network and gsm. International Journal of Advnced Research in Computer Engineering \& Technology. 2015 Jan;4(1).

[12] RenukaChuimurkar M, Bagdi V. Smart Surveillance Security \&Monitoring System Using Raspberry PI and PIR Sensor. International Journal of Scientific Engineering and Applied Science (IJSEAS). 2016 Jan;2(1):1-4.

[13] G. J. H. R. Ani and V. R. Amya, "Efficient Camera Based Monitoring and Security System using Raspberry Pi," vol. 4, no. 19, pp. 3677-3680, 2016.

[14] Tarun. Raspberry Pi Node Red [Internet]. Hackster.io. 2017 [cited 2018Dec15]. Available from: https://www.hackster.io/tarun/raspberry-pi-node-red-463cc3

[15] A. H. H. Basri, S. N. Ibrahim, N. A. Malik and A. L. Asnawi, "Integrated Surveillance System with Mobile Application," 2018 7th International Conference on Computer and Communication Engineering (ICCCE), Kuala Lumpur, 2018, pp. 218-222.

\section{BIOGRAPHIES OF AUTHORS}
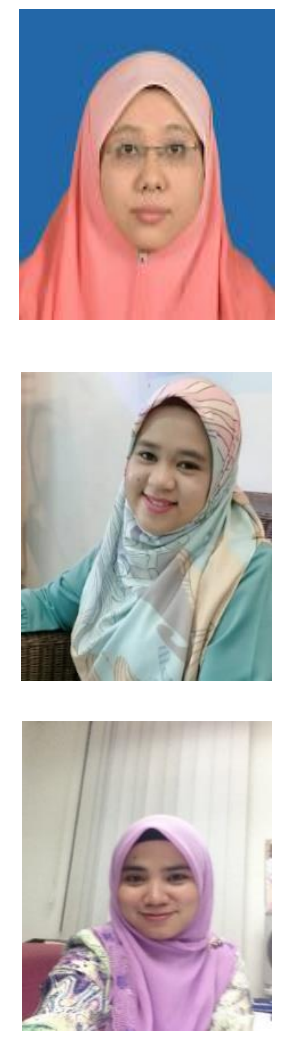

S.Noorjannah Ibrahim currently an Assistant Professor in the Department of Electrical and Computer Engineering, Faculty of Engineering, International Islamic University Malaysia. She has a Ph.D. in Electrical and Computer Engineering from the University of Canterbury, New Zealand (2012). She obtained her M.Sc in Microelectronic Systems and Telecommunications from the University of Liverpool (2003), and her Bachelor degree in Electronics(Medical Electronics) from Universiti Teknologi Malaysia (UTM). Her current research interests include wireless technology and Internet of Things (IoT), wireless communications in biomedical devices. She is a Senior Member of IEEE, Volunteer for IEEE Electron Devices Society (Malaysia Chapter), and a registered member for BEM and IEM.

A.H Hasan Basri graduated in Bachelor of Telecommunication Engineering from IIUM (2018). One of the her major achievement was developing the Integrated Surveillance System with Mobile Application and the project was awarded as the best FYP award in IoT category by IEEE Computer Society (Malaysia Chapter) under supervision of Dr.S. Noorjannah Ibrahim.

A.L. Asnawi is currently an Assistant Professor in Electrical and Computer Dept, Faculty of Engineering, International Islamic University Malaysia. She received her Phd from School of Electronics and Computer Science, University of Southampton, UK, in 2012. She obtained her M. Eng in Communication and Computer Engineering from University Kebangsaan Malaysia (UKM), and her Bachelor degree (Computer and Information Engineering) from International Islamic University Malaysia (IIUM). Her current research interests include wireless communication, software defined radio, software engineering, empirical software engineering, Agile methods and software processes. She is an active member of IEEE, IEEE Computer Society, a registered member for BEM and IEM. 